\title{
Figurer les entre-deux migratoires
}

Pratiques cartographiques expérimentales entre chercheurs, artistes et voyageurs

Sarah Mekdjian, Anne-Laure Amilhat-Szary, Marie Moreau, Gladeema Nasruddin, Mabeye Deme, Lauriane Houbey et Coralie Guillemin

\section{OpenEdition}

Édition électronique

URL : http://journals.openedition.org/cdg/790

DOI : $10.4000 /$ cdg. 790

ISSN : 2107-7266

Éditeur

UMR 245 - CESSMA

Référence électronique

Sarah Mekdjian, Anne-Laure Amilhat-Szary, Marie Moreau, Gladeema Nasruddin, Mabeye Deme, Lauriane Houbey et Coralie Guillemin, «Figurer les entre-deux migratoires », Carnets de géographes [En ligne], 7 | 2014, mis en ligne le 01 décembre 2014, consulté le 19 avril 2019. URL : http:// journals.openedition.org/cdg/790; DOI : 10.4000/cdg.790

Ce document a été généré automatiquement le 19 avril 2019.

La revue Carnets de géographes est mise à disposition selon les termes de la Licence Creative Commons Attribution - Pas d'Utilisation Commerciale - Pas de Modification 4.0 International. 


\title{
Figurer les entre-deux migratoires
}

Pratiques cartographiques expérimentales entre chercheurs, artistes et voyageurs

\author{
Sarah Mekdjian, Anne-Laure Amilhat-Szary, Marie Moreau, Gladeema \\ Nasruddin, Mabeye Deme, Lauriane Houbey et Coralie Guillemin
}

Nous tenons à remercier vivement les relecteurs-rices de cet article pour les conseils donnés.

Des voyageurs ${ }^{1}$, en situation de demande d'asile ${ }^{2}$ ou ayant obtenu le statut de réfugiés ${ }^{3}$, des chercheurs, des travailleurs associatifs, des artistes se sont réunis deux fois par semaine, à Grenoble, pendant deux mois, entre mai et juin 2013, pour co-produire des cartes de voyages migratoires. Ces cartes figurent des fragments de souvenirs, des traces d'expériences des voyages et des franchissements frontaliers vécus par douze demandeurs d'asile ou réfugiés, qui habitent actuellement à Grenoble.

Entre art et sciences humaines, entre intentions esthétiques et scientifiques, les modalités d'expression cartographique comprennent des cartes dessinées sur papier à partir d'une légende discutée et utilisée en commun, des cartes mentales individuelles dessinées puis brodées sur tissu, la réalisation d'une carte en argile, la production de pièces sonores à partir des souvenirs des bruits entendus pendant les voyages. A partir de ces différentes pratiques cartographiques, il s'agissait d'ouvrir un temps d'échanges et de création entre artistes, chercheurs et voyageurs, hors des cadres habituels des techniques d'enquête et d'interview, hors des cadres administratifs d'enregistrement des demandes d'asile et de droit de séjour.

3 La cartographie désigne ici une pratique de décentrement narratif; l'originalité des cartes produites n'est pas relative aux avancées des techniques de visualisation mobilisées, ni de la sémiologie graphique choisie. Les narrations cartographiques engagées permettent de créer un décentrement par rapport aux catégories usuelles de la recherche et des administrations.

Dans un article paru en 2012 dans la même rubrique des Carnets de Géographes, Nathalie Bernardie-Tahir et Camille Schmoll s'interrogeaient sur « la voix des chercheur(-e)s et la parole des migrants » à partir d'une analyse critique et réflexive d'un terrain mené à Malte. Les auteures appelaient à une nécessaire «triangulation des sources et des 
techniques de recueil des données» (2012, p. 11), pour tenter d'approcher et d'analyser des expériences sociales complexes, dont l'entretien ou l'interview avec les individus concernés n'auraient pu rendre compte. Réfléchir à un renouvellement des méthodes de recherche, à partir d'outils de médiation participatifs s'inscrit dans une perspective à la fois scientifique et politique. Comment co-construire un terrain et des données de recherche avec les individus concernés par les problèmes étudiés, dans la perspective de produire des "discours sur" mais aussi d'"agir avec», de "lutter contre», de "s'engager pour»? L'engagement dans une démarche participative, autour du geste cartographique, est un axe de «triangulation " possible dans l'entre-deux des relations inter-individuelles, mais aussi entre art et science.

5 Ainsi, l'expérience des ateliers a engagé une réflexion sur plusieurs registres d'entredeux: l'entre-deux du voyage et des espaces de transit vécu et raconté par les participants ; la cartographie, comme outil de médiation, entre-deux méthodologique, mis au service des relations tissées entre les participants. La figure de l'entre-deux sert ainsi à la fois à désigner notre objet de recherche et notre méthode : l'espace-temps des voyages migratoires, et le défi méthodologique qui a consisté à créer un cadre d'échanges entre des individus caractérisés par plusieurs écarts, linguistiques, discursifs, sociaux, administratifs.

6 Comme les ateliers de cartographie, ce texte est polyphonique et expérimental. A défaut de théorisation définitive, nous proposons d'ouvrir en acte un espace d'échanges à plusieurs voix sur cette expérience en cours. Plutôt que de parler au nom des migrants, des artistes ou des chercheurs engagés dans ce projet, plusieurs participant.e.s ont saisi l'opportunité de s'exprimer en leur propre nom. Les échanges entre les participant.e.s ne sont pas rapportés sous forme de citations. Pendant les ateliers, les voyageurs n'ont pas été interviewés par les chercheurs ni écoutés à partir d'une méthodologie de recueil de récit de vie. Chaque participant s'est exprimé dans le cadre d'un travail cartographique proposé par les chercheurs et les artistes et discuté en commun.

7 Après avoir exposé des propositions cartographiques mises en œuvre, Sarah Mekdjian et Anne-Laure Amilhat-Szary, enseignantes-chercheuses en géographie, expliquent les objectifs scientifiques des ateliers. Puis Marie Moreau, artiste plasticienne, revient sur ses propres intentions de participer à cette expérience, en proposant une réflexion sur la carte comme entre-deux d'un art relationnel et géographique. Lauriane Houbey, artiste plasticienne, présente la pièce sonore qu'elle a réalisée. Mabeye Deme, photographe des ateliers, évoque sa conception des entre-deux relationnels tissés avec les participants. Enfin, Gladeema Nasrudddin, qui a souhaité être présenté en tant que participant des ateliers et demandeur d'asile, commente une des cartes qu'il a réalisée, en s'adressant directement aux lecteurs de l'article. Nous rendons compte chacun.e à notre manière ${ }^{4} \mathrm{de}$ cette expérience. La réflexion croisée que nous proposons transcrit l'hétérogénéité constitutive de nos intentions et de nos points de vue.

\section{Constitution des ateliers}

Pendant deux mois, de mai à juin 2013, à la Maison des Associations à Grenoble, chercheurs, artistes, travailleurs associatifs et voyageurs ${ }^{5}$ se sont rencontrés deux fois par semaine. Chaque séance durait en moyenne deux heures. 


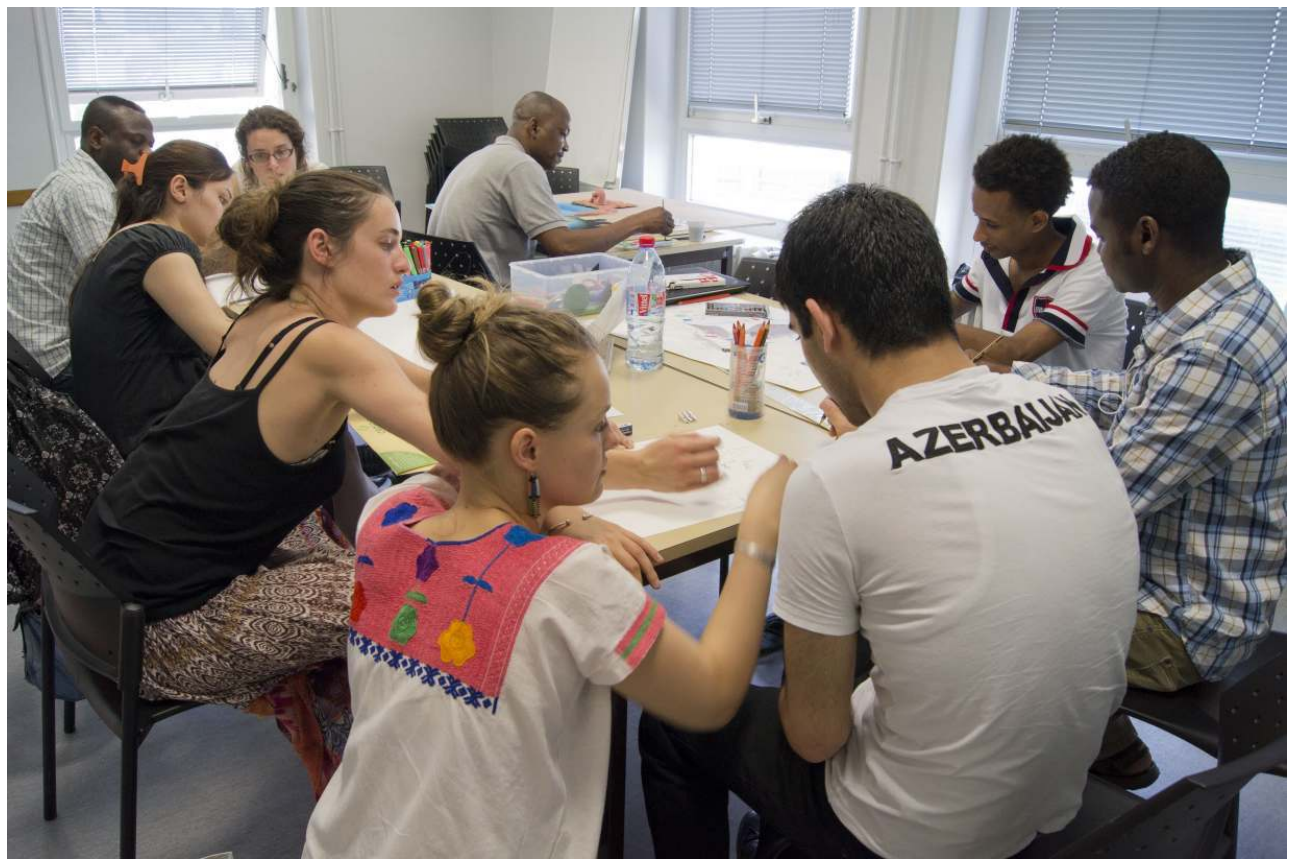

Photographie : Mabeye Deme, atelier de cartographie, Grenoble, juin 2013.

9 A la Maison des Associations, les ateliers ont été accueillis dans les locaux de l'association Accueil Demandeurs d'Asile (ADA) ${ }^{6}$.

10 Avec la mise à disposition de ses locaux, l'ADA nous a aussi permis d'entrer en contact avec des personnes en situation de demande d'asile ou ayant obtenu le statut de réfugié. Douze personnes ont répondu à l'invitation de Sarah Mekdjian de participer, bénévolement, à des ateliers participatifs et expérimentaux de cartographie. Ces personnes parlent des dizaines de langues différentes, ont habité et parcouru de très nombreux pays, ont été ou sont encore de nationalité soudanaise, érythréenne, arménienne, congolaise, guinéenne, algérienne, azérie et afghane. Certain-e-s étaient à l'époque de nos rencontres demandeur-se-s d'asile, réfugié-e-s, d'autres, exclu-e-s des statuts et circuits administratifs.

\section{Quatre propositions de pratiques cartographiques entre art et sciences humaines}

Quatre propositions de pratiques cartographiques ont été développées au fil des séances, pour figurer les espaces-temps parcourus jusqu'à Grenoble et au sein de cette ville par les personnes rencontrées.

12 1-La proposition crée par Sarah Mekdjian et Anne-Laure Amilhat-Szary, intitulée «Les légendes $d u$ voyage ", a consisté à construire avec les voyageurs une légende commune, à partir de leurs souvenirs des expériences vécues pendant le voyage. Après généralisation (treize termes à visualiser ont été retenus collectivement), la codification a été matérialisée par des gommettes de couleurs et de formes géométriques différentes. 
Figure 2. "Les légendes du voyage »

Photographie : Mabeye Deme, atelier de cartographie, Grenoble, juin 2013

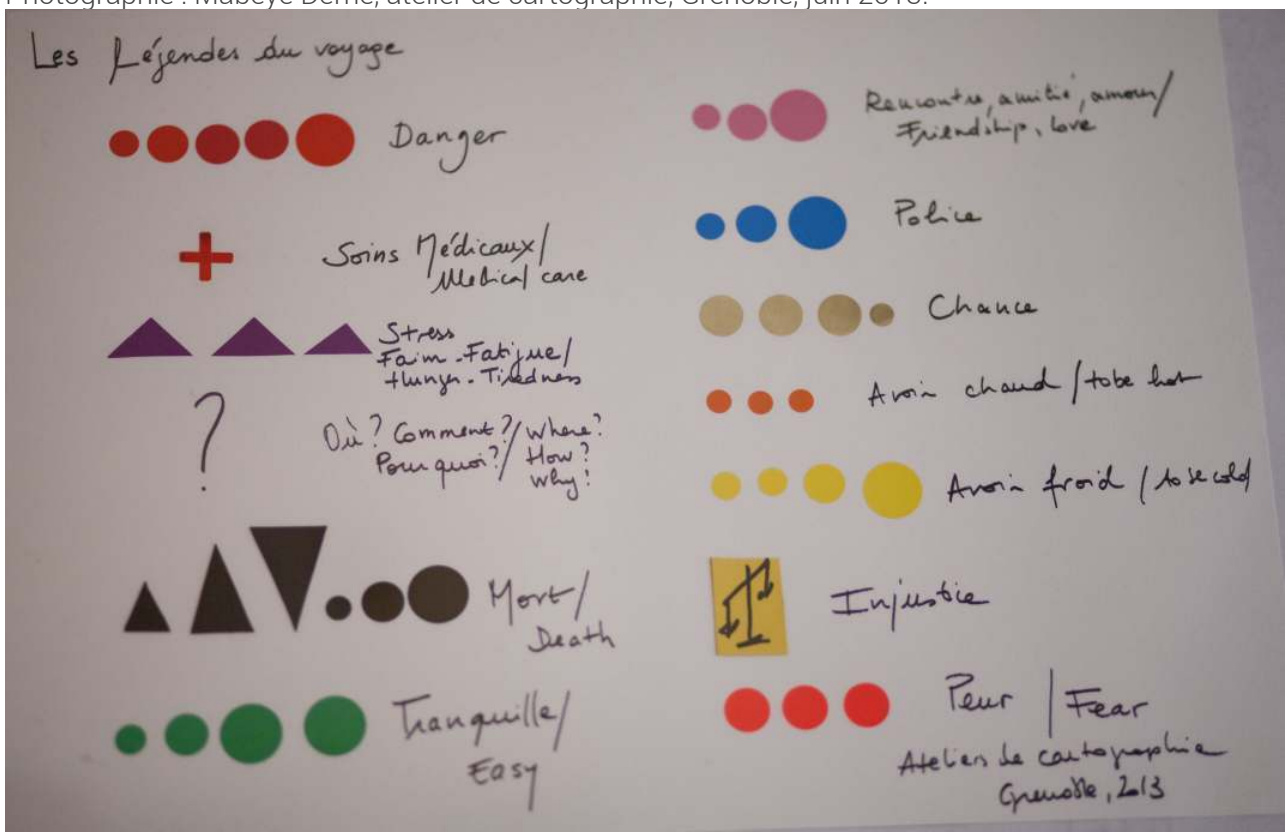

Chaque participant a ensuite dessiné plusieurs cartes individuelles en mobilisant la légende, un travail participatif qui va au-delà des cartes mentales que l'on présente traditionnellement, dans la lignée des travaux de K Lynch (1998).

Figure 3. «Déplacements depuis le Soudan »

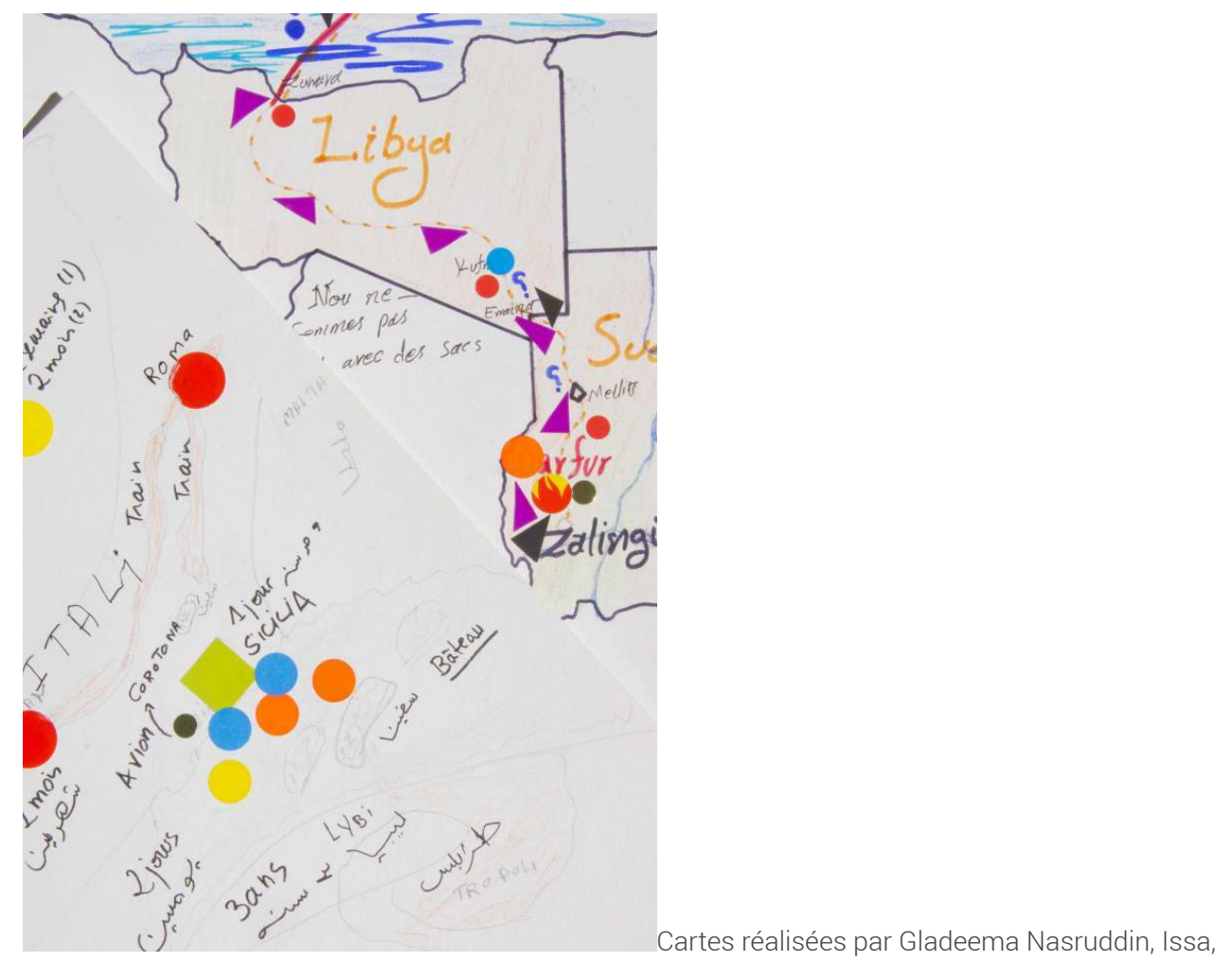

Sarah Mekdjian et Coralie Guillemin, atelier de cartographie, Grenoble, juin 2013. Photographie:

Mabeye Deme 
2-Marie Moreau a demandé aux voyageurs de dessiner individuellement leurs voyages sur de grandes nappes blanches, qu'elle a ensuite brodées en partie. Ce dispositif est proche de la cartographie mentale. L'œuvre réalisée intitulée "Atlaslocal» (2013) est composée des cartes dessinées et brodées avec les participants des ateliers de cartographie et d'autres personnes rencontrées dans des centres sociaux grenoblois.

Figure 4. « Amour », extrait

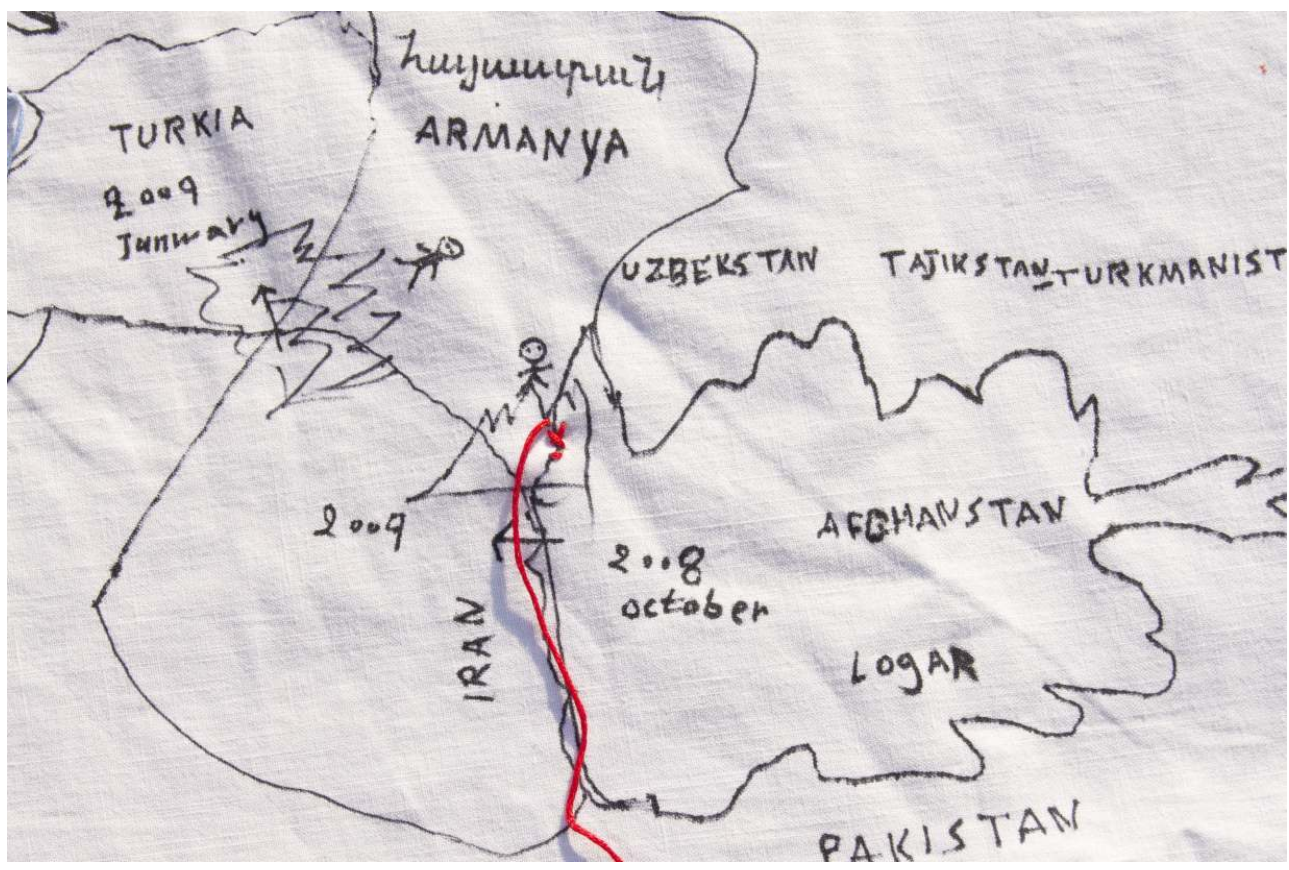

« Atlaslocal », carte réalisée par Halimjan et Marie Moreau, tissu, feutre, fil, Grenoble, atelier de cartographie, juin 2013. Photographie : Mabeye Deme

\section{La carte comme entre-deux et entremise d'un art relationnel et géographique}

Texte de Marie Moreau, artiste plasticienne, association ex.C.es.

Quand j'ai commencé ce projet d'Atlaslocal, j'imaginais des cartographies subjectives représentant des itinéraires inconnus, des chemins empruntés, braconnés, inventés. Une géographie de «lignes d'erre » (Deligny, 1975 [2007]). J'avais entendu ce récit d'une travailleuse sociale du Fournil (table d'hôtes associative pour les désargentés) ; elle racontait qu'à midi, autour des tables, depuis quelques années et par vagues, les hôtes demandaient des stylos pour dessiner leur trajet, leur point de départ et de "chute ». Les cartes-dessins représentaient les abords de la table d'hôte, des villes inconnues ou récemment citées aux « actualités ». J'ai demandé à voir ces dessins, ils n'avaient pas été conservés, ils étaient l'outil d'un moment, un service rendu à la rencontre. Cet atlas virtuel issu des nécessités relationnelles m'a poursuivi jusqu'à ce que je décide de le reprendre.

Je me suis rendue à cette table d'hôte, puis plus tard dans d'autres lieux d'accueil de jour. J'ai ensuite poursuivi mon travail à l'Atelier de cartographie participatif et expérimental créé avec l'Université de Grenoble et accueilli dans les locaux l'association Accueil Demandeurs d'Asile. J'ai redécouvert une fois de plus à quel point ces lieux d'accueil sont des relais, des auberges à la croisée des chemins, des refuges où les histoires se racontent par bribes, par fragments. Dans ces lieux de passages, il y a quotidiennement jusqu'à une centaine de personnes qui viennent se 
réfugier, se doucher, manger, parler, déposer leurs bagages, constituer leurs dossiers administratifs... Ce sont des lieux où l'on attend, où l'on passe du temps, pour ne pas être seul.

C'est là, dans ces relais, en m'y installant que j'ai rencontré Alpha, par son dessin. Il a cartographié les rues, les bâtiments autour de l'association sur un set de table. Son dessin était tellement intrigant, dans sa facture, ses repères, sa géographie, que je lui ai demandé s'il pouvait me dessiner la ville, plus loin encore, après l'arrêt Malherbe et de l'autre côté, après l'arrêt Albert 1er de Belgique en collant un autre set de table, comme ça à plusieurs reprises, jusqu'à ce qu'il s'en aille. Je l'ai retrouvé plus tard dans un autre lieu d'accueil, un matin. Je nappais les tables dehors, dedans, avec des draps blancs comme une page blanche. J'invitais les gens à dessiner leurs trajectoires et leur géographie intérieure ${ }^{2}$. Je me suis installée autour d'une de ses nappes et Alpha a dessiné le monde en commençant par la Corne de l'Afrique. Comme il y avait des gens autour, ce dessin est devenu le monde revu et corrigé par Alpha, Petru, Brahim et Aslia. Chacun dessinait depuis son expérience du territoire. Dans ces traits, un monde apparaît par lequel il devient possible de se frayer un chemin, de passer outre le mur de la langue.

Tous ces dessins m'ont fait penser aux tapisseries ou mosaïques qui imageaient les péripéties des héros antiques. Voilà pourquoi les cartes sont brodées. Les cartes sont devenues une collection en cours, un atlas local à colporter.

16 3-Lauriane Houbey a travaillé avec les voyageurs à la constitution de cartographies sonores, qui donnent à entendre les souvenirs des bruits entendus pendant les voyages, et souvent associés à des milieux spécifiques (montagnes, déserts, mers...). Son travail s'est appuyé notamment sur le commentaire des cartes dessinées avec Sarah Mekdjian, AnneLaure Amilhat-Szary et Marie Moreau. L'œuvre sonore réalisée, intitulée «D'ici là le milieu », est présentée par l'artiste dans la figure 5. 

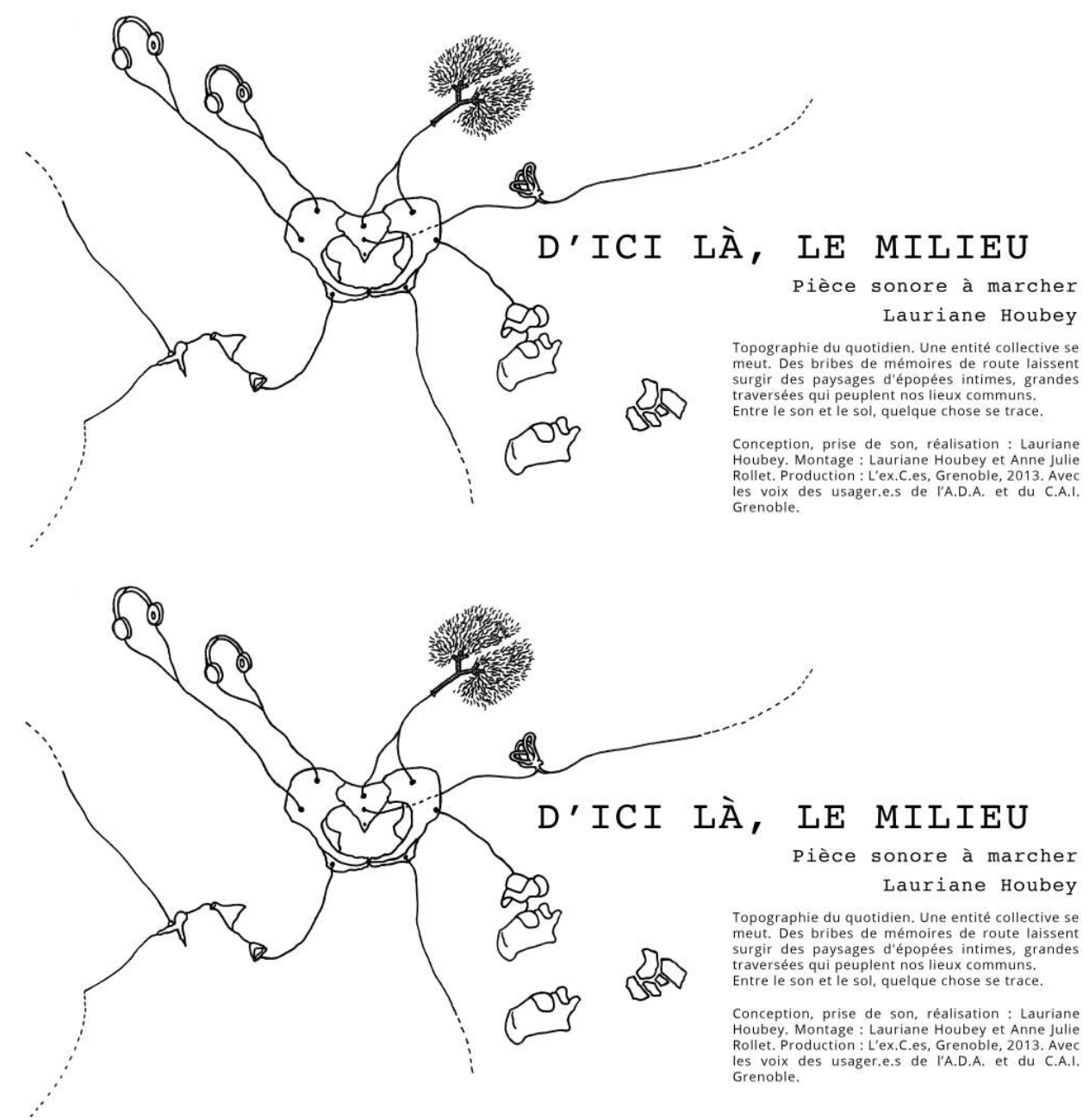

Texte et dessin de Lauriane Houbey, à partir des ateliers de cartographie, Grenoble, septembre 2013.

4-Fabien Fischer, dans son œuvre sonore intitulée «Ici, pas encore », a enregistré des conversations menées entre les participants, portant sur la vie à Grenoble, les lieux fréquentés, les expériences locales.

Mabeye Deme a suivi les ateliers en photographie, tandis que Coralie Guillemin était chargée de leur organisation, notamment de la mobilisation individuelle des participants tout au long du projet.

Les cartes, dessinées, brodées ou enregistrées, ont constitué des outils de médiation dans les relations tissées entre les participants du projet. Au total, 25 cartes sur papier, 14 cartes dessinées et brodées sur tissu, une carte en relief réalisée en argile et plusieurs heures d'enregistrements sonores ont été co-produites.

Après avoir exposé les enjeux scientifiques et éthiques d'un projet de recherche participatif qui porte sur les voyages migratoires, nous verrons en quoi la carte peut être un outil méthodologique de médiation mis au service des relations inter-individuelles et collectives. 


\section{Intentions scientifiques des ateliers}

\section{(Géo)graphies des voyages migratoires}

21 Les recherches en sciences humaines sur les migrations internationales s'intéressent aux circulations et aux territoires migratoires, en particulier les espaces parcourus pendant les voyages migratoires, de plus en plus surveillés et contrôlés. Les travaux d'Alain Tarrius (Tarrius, Missaoui, Qacha, 2013), de Michel Agier (2011), d'olivier Pliez (2011) ou encore de Laurent Faret (Faret, 2002; Faret, Cortes, 2009) sur la géographie et l'anthropologie des parcours migratoires mais aussi les recherches sur les économies du passage, ont contribué à faire connaitre l'importance des espaces de transit. Plus qu'un passage, le voyage migratoire est un espace-temps spécifique, plus ou moins étendu, qui engage des pratiques socio-spatiales spécifiques. La littérature sur les migrations africaines vers l'Europe en particulier montre combien le voyage est central dans les expériences migratoires (Arab, 2007 ; Bennafla, Peraldi, 2008 ; Pian, 2009 ; Pliez, 2010).

Sur de nombreuses cartes migratoires, qui paraissent notamment dans les médias, les espaces parcourus par les migrants pendant leurs voyages sont souvent « lissés ", selon la terminologie utilisée par Armelle Choplin et Olivier Pliez (2011) au sujet des cartes de l'espace migratoire transsaharien: «la plupart des représentations cartographiques choisies aboutissent à la vision d'un espace migratoire « lisse », c'est-à-dire où le trait de dessin continu de quelques routes migratoires occulte toutes les « aspérités »-spatiales et temporelles d'ordre politique, policier, pécuniaire...- qui jalonnent les itinéraires empruntés par les migrants » (Pliez, Chopplin, 2011). En réaction à la figuration d'espaces « lissés ", des chercheurs tentent de produire des cartes où apparaissent les expériences vécues pendant les déplacements et les difficultés à franchir des frontières de plus en plus surveillées. L'Atlas des migrants en Europe publié par le collectif Migreurop (2009, 2012), mais aussi des productions de contre-cartographie sur les franchissements frontaliers, entre art, science et activisme, se multiplient ${ }^{7}$.

Les expériences de franchissements frontaliers sont en effet des événements très significatifs dans les histoires migratoires individuelles. Pour les personnes qui n'ont pas de droit de séjour, la frontière parcourue s'étend jusque dans les espaces dits d'accueil. Ainsi, à Grenoble, mais cela pourrait être ailleurs dans de nombreuses villes européennes, des voyageurs voyagent encore... Les lieux associatifs, le Point d'eau, pour se laver, le Fournil, pour manger, l'association Accueil Demandeurs d'Asile à la Maison des Associations, pour les démarches administratives... sont les repères instables d'une ville qui ne cesse de se dérober sous leurs pas. Le droit de séjour est suspendu à la Préfecture de l'Isère, lieu des espoirs/désespoir.

24 L'enjeu de cartographier les aspérités socio-spatiales de ces espaces migratoires apparaît à la fois comme scientifique, artistique et politique. Nous avons ainsi tenté d'explorer des modes de figuration créative pour envisager différentes formes de « cartes d'itinéraires » (Besse, 2010), co-produites par les voyageurs eux-mêmes, au risque de bousculer les conventions afférents au modèle cartographique. Les cartes d'itinéraires sont définies par Jean-Marc Besse comme des cartes qui «fourni[ssen]t une représentation du territoire dans laquelle celui-ci n'est pas considéré indépendamment des pratiques qui s'y déploient [...] mais au contraire défini dans sa structure même par les engagements pratiques de ceux qui y inscrivent leurs déambulations » (Besse, 2010, p. 7). 


\section{Les cartes, outils participatifs de médiation entre chercheurs, artistes, voyageurs} ont autorisé des échanges indisciplinaires ${ }^{8}$ fructueux, entre art et sciences. Intentions scientifiques et esthétiques se sont complétées à partir de la carte. Après D. Cosgrove (1999) et D. Wood (1992, 2010), A. Volvey (2007) et G. Tiberghien (2010) ont montré combien la cartographie était un procédé de plus en plus partagé par la science, principalement géographique, et l'art contemporain. Dans ce cadre, la frontière et son franchissement apparaissent comme des "figures fertiles" (Grison, 2002 ; Rogoff, 2000 ; Amilhat-Szary, 2012) pour analyser les processus à l'œuvre dans cet entre-deux de la connaissance. Les propositions cartographiques artistiques -voire artivistes- développées par Marie Moreau, Lauriane Houbey et Fabien Fischer ont détourné en partie les conventions cartographiques scientifiques. Les cartes sonores, par exemple, introduisent des données sensorielles aux relevés d'expériences et à la figuration de l'espace, tandis que la matérialité des cartes en tissu brodé, co-produites par Marie Moreau et les voyageurs, engage une réflexion sur les fonctions de la carte comme objet, et non seulement comme «iconotexte» (Cosgrove, 2001, p. 148). Les pratiques cartographiques mises en œuvre par les artistes ont nourri des intentions esthétiques propres à chacun d'entre-eux, mais aussi la portée critique et scientifique du projet pensé par les chercheurs.

Mabeye Deme, photographe du projet, s'est servi de la photographie, en plus de la cartographie, comme outil de médiation pour élaborer des relations avec les participants. Les photographies réalisées servent aussi à médiatiser le travail produit pendant les ateliers auprès du public. 


\section{La carte et l'appareil photographique au service de la relation, texte de Mabeye Deme, photographe}

Dans l'entre-deux, un appareil photo. Dans l'entre-nous un désir de s'observer, de se déchiffrer, de se rencontrer... L'image est un monde indépendant, un espace de rencontre sans frontières, que l'on habite ensemble et qui documente autant le visible que le non-visible. A mesure que la confiance s'installe et que nous baissons progressivement nos gardes, nous livrons nos gestes, nos postures, nos mystères au regard de l'autre. Je photographie leurs paroles pendant qu'ils regardent mes silences. Mon regard voyage entre ces cartes en train de se faire, je ne cherche pas à me faire oublier. Je me laisse guider par ce « nous », je navigue dans le flou lorsque, soudain, je vois.

Figure 6. « Alishum »

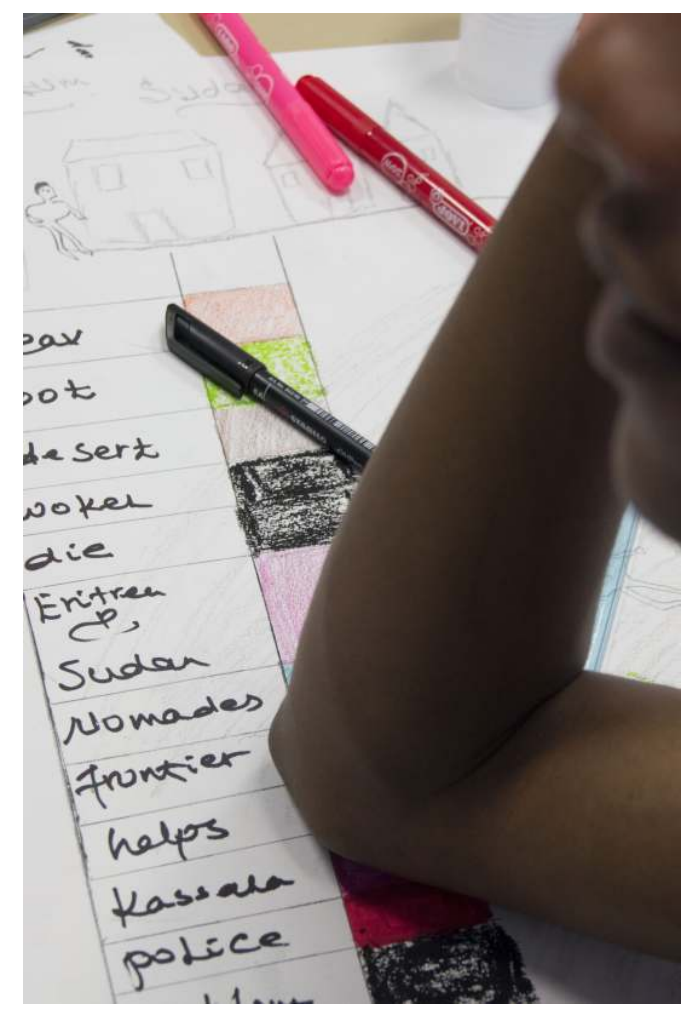

Photographie : Mabeye Deme, atelier de cartographie, Grenoble, juin 2013.

\section{Des méthodes participatives critiques?}

La cartographie en tant qu'outil participatif est une manière alternative de co-produire des données ; néanmoins elle n'est pas sans poser plusieurs difficultés éthiques. Ainsi, la participation n'est jamais dégagée de la question de la représentation; elle est toujours produite dans un cadre ensemble d'intérêts et de relations de pouvoir. Ainsi, si la participation permet en partie de remettre en cause la posture d'autorité des chercheurs à partir de laquelle ils représentent, au sens où ils parlent pour les personnes qu'ils étudient, la participation n'est jamais «directe » ni transparente. Elle s'élabore à partir d'outils de médiation et se trouve nécessairement prise dans des rapports de représentation, et en conséquence de pouvoir. On retrouve ici les deux sens de la 
représentation : représenter au sens de "parler au nom de/parler pour» et au sens de " re-présenter ", «mettre en scène ». Si la pratique cartographique a été appropriée par chaque participant pour s'exprimer en leur nom, les cartes produites constituent des formes de re-présentation, au sens de mise en scène "performati[ve] et configurant[e] » (Volvey, 2007, p. 7) du monde.

Dans le registre de la représentation politique, la possibilité pour les participants de se saisir de la cartographie pour "parler en leur nom» ne va pas de soi. G. S. Spivak, dans son célèbre texte « Les subalternes peuvent-elles parler?» (2006), élabore une critique de l'idéal d'une participation émancipatrice. Prétendre à partir d'un point de vue intellectuel que les individus en situation de "subalternes " peuvent clairement parler pour eux/ elles-mêmes, c'est-à-dire concevoir les individus comme des "sujets", revient à nier l'idéologie qui sous-tend la notion même de "parole claire » et plus encore de "sujet ", entendu comme "sujet souverain». En acceptant que la participation n'est jamais directe, ni claire, toujours médiatisée et prise dans des rapports de représentation, il s'agit de réfléchir aux modalités de la représentation et aux intérêts qu'elles servent.

31 Au sein du collectif qui a émergé pendant les ateliers, les chercheurs et les artistes ont assumé un rôle déclencheur, tant en termes d'organisation des modalités d'atelier que de certains éléments de contenu figurés sur les cartes. Par ailleurs, la question du cadre institutionnel des ateliers, qui est aussi le cadre de travail partagé par les participants, a été très discutée entre tous les participants, particulièrement quand les résultats ont pu être exposés pour la première fois ${ }^{9}$ et que la communication autour de cette exposition ${ }^{10} \mathrm{a}$ rappelé l'existence de l'Union Européenne, commanditaire indirecte du travail par le biais de ses financements ${ }^{11}$. Ce lien entre intention critique et positionnement institutionnel mérite bien évidemment approfondissement. La sélection de ce travail au sein du dispositif Art-Sciences-Technologie "Anti-Atlas des frontières » et de son exposition Au Musée des Tapisseries d'Aix-en-Provence du 1er octobre au 3 novembre 2013 ouvrira sans nul doute une nouvelle phase de problématisation pour nous.

Il est ressorti toutefois que si les participants avaient surtout travaillé pour eux-mêmes, dans une dynamique très cathartique, ils étaient particulièrement demandeurs que les cartes produites circulent, de façon à pouvoir montrer leur travail et s'en servir pour engager des relations avec le public. Le texte écrit par Gladeema Nasruddin, présenté ciaprès, est adressé directement aux lecteurs de cet article: il exprime un discours politique sur les situations sociales et politiques vécues par les demandeurs d'asile. Le texte vient aussi en accompagnement des photographies d'une des cartes que Gladeema Nasruddin a réalisée pendant les ateliers, intitulée «The world is stopping us/Le monde nous empêche d'avancer » (figures 7 et 8). La situation exprimée à travers la carte est aussi au fondement de l'engagement de Gladeema Nasruddin au sein des ateliers. 
Figure 7. « The world is stopping us/Le monde nous empêche d'avancer ». Vue 1
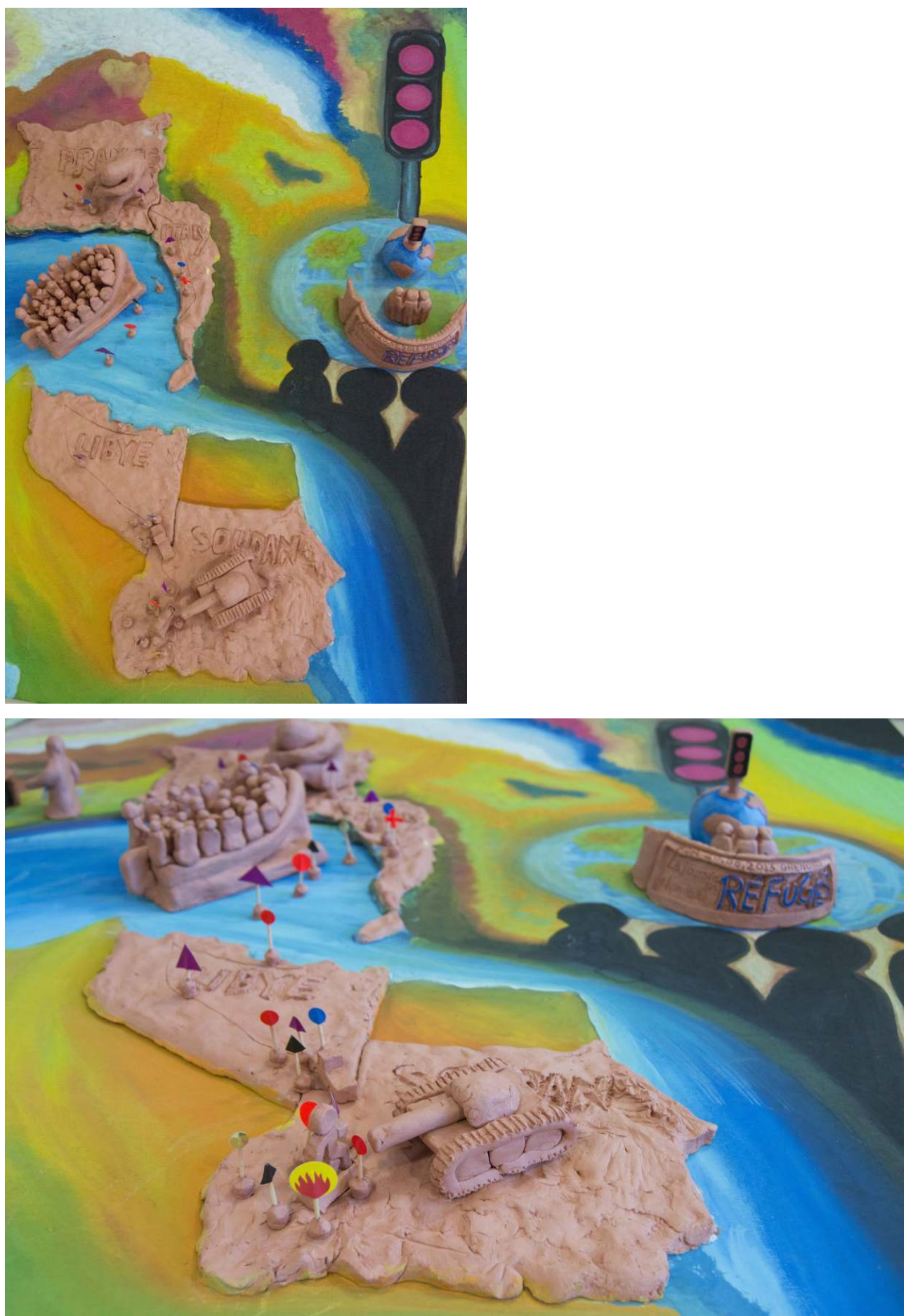

CARTE RÉALISÉE PAR GLADEEMA NASRUdDIN, ARgILE, BOIS, PEINTURE, GRENOBLE, ATELIER DE CARTOgRAPHIE, JUIN 2013, PHOTOgRAPHIES : MABEYE DEME 
Figure 8. « The world is stopping us/Le monde nous empêche d'avancer ». Vue 2

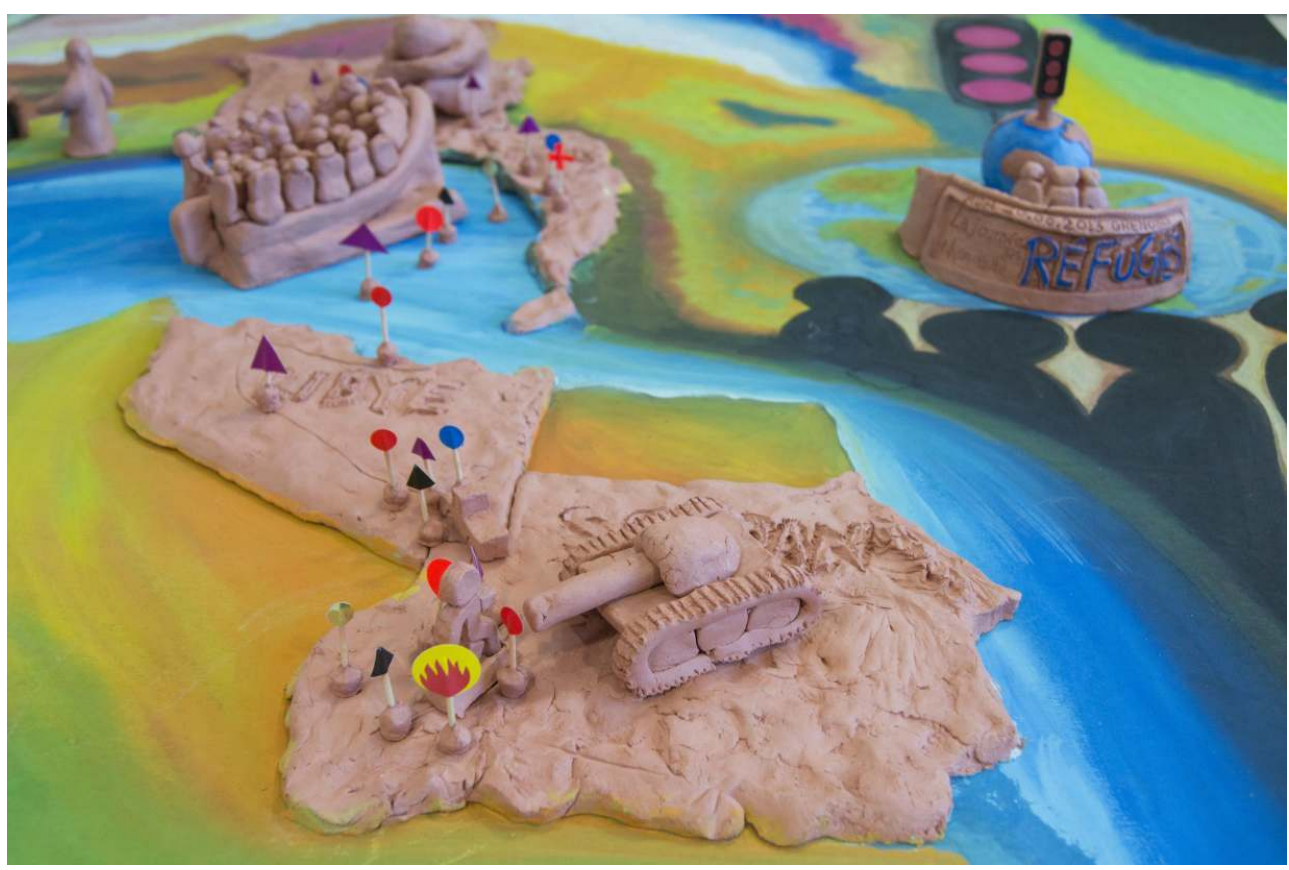

Carte réalisée par Gladeema Nasruddin, argile, bois, peinture, Grenoble, atelier de cartographie, juin 2013, photographies : Mabeye Deme

The world is stopping us/Le monde nous empêche d'avancer

Texte et carte de Gladeema Nasruddin, participant aux ateliers et demandeur d'asile ; texte original en anglais de Gladeema Nasruddin

When you lose the choice of returning to your country of origin because the specter of death is waiting there for you, while living in another country that does not accept you and you have neither documents nor a visa to travel to a third destination, then you feel as if the whole world stands against you. This feeling makes you unable to breathe most of the time. And it makes you strongly hate all the laws that stop you. But do not worry, because this will never happen to you if you are not an asylum seeker.

Still thousands of asylum seekers in Europe continue to flee from a country to another in search of protection and stability. They only trust the human traffickers because they are the only ones who are able to give them the opportunity to start another life in a different country.

Some of the common European laws such as the Dublin Convention 2, in addition with a lot of arbitrary actions that apply in some European countries, have misrepresented many of the asylum seekers as criminals wanted by the police and made them a burden on citizens and governments. These laws have also turn thousands of asylum seekers into victims of drug addiction.

Version originale en anglais, Gladeema Nasruddin 
Quand vous n'avez plus le choix de rentrer dans votre pays d'origine en raison de la mort probable qui vous y attend, lorsque vous vivez dans un pays qui ne vous accepte pas et que vous n'avez ni papiers, ni visas pour tenter une autre destination, vous avez alors l'impression que le monde entier est contre vous. Cette impression vous empêche de respirer quasiment tout le temps; elle vous pousse aussi à haïr les lois qui vous obligent à cette immobilité. Mais ne vous inquiétez pas, cela ne vous arrivera jamais si vous n'êtes pas demandeur d'asile.

Des milliers de demandeurs d'asile en Europe continuent encore à fuir de pays en pays pour trouver une protection et une stabilité. Ils ne font confiance qu'aux passeurs, car ce sont les seuls qui puissent leur donner la possibilité de recommencer une autre vie dans un nouveau pays.

Certaines lois communautaires de l'Union Européenne, notamment le Règlement Dublin II, avec de nombreuses autres décisions arbitraires appliquées par les pays européens, présentent les demandeurs d'asile à tort comme des criminels recherchés par la police et en font des fardeaux pour les citoyens et les gouvernements des pays qu'ils habitent. Ces lois ont aussi contribué à ce que des milliers de demandeurs d'asile deviennent victimes des addictions aux drogues.

\section{Traduction française, Sarah Mekdjian}

\section{Conclusion}

L'expérience des ateliers de cartographie participatifs et expérimentaux organisés à Grenoble a engagé plusieurs niveaux d'entre-deux.

A travers ce projet, nous questionnons les voyages migratoires, depuis une médiation alternative des récits exprimés par les personnes qui en ont fait l'expérience. Ce travail permet aussi d'étudier la figure de l'entre-deux d'un point de vue méthodologique, c'està-dire les conditions de mises en relation des acteurs d'un projet de recherche et de création (artistes, chercheurs, voyageurs). Comment communiquer entre les chercheurs, les artistes et les personnes invitées en raison de leurs parcours migratoires? Les écarts entre ces acteurs sont d'ordre linguistiques, discursifs, sociaux, administratifs, tandis que plusieurs intentions expliquent l'engagement de chacun dans le projet. Les ateliers ont été perçus comme des opportunités à saisir pour participer aux débats scientifiques et politiques sur les migrations et les frontières, pour explorer des imaginaires et des gestes créatifs ou encore pour s'occuper pendant le temps d'attente, souvent très long et incertain, des décisions administratives.

La cartographie, appropriée par tous selon des modalités diverses, a servi d'outil de médiation dans les relations entre les différents acteurs des ateliers et avec le public. Plusieurs types de pratiques cartographiques participatives ont été proposées par les artistes et les chercheurs, puis mises en œuvre. Ces performances cartographiques ont permis d'envisager des possibles, créer des chemins de traverses et des "lignes d'erre » (Deligny, 2007), élaborer des narrations alternatives, et ouvrir des imaginaires.

36 La réalisation de ces cartes, visuelles et sonores, a permis en partie de subvertir l'injonction administrative de produire des récits chronologiques et linéaires particulièrement normatifs, contraignants et assujettissants (Fassin, Kobelinsky, 2012). 
Nous souhaitions éviter de reconduire ces conditions normatives dans des interviews ou des entretiens, à partir desquels nous aurions parler pour les migrants.

Raconter des souvenirs de voyages par des pratiques cartographiques alternatives et artistiques a constitué un cadre critique et créatif d'échanges.

La participation à travers la cartographie n'évacue pas les questions relatives au pouvoir et à la représentation dans le processus de constitution des données de recherche. Les pratiques participatives mises en œuvre, notamment le fait de co-signer cet article avec un des participants des ateliers, permettent non pas de «donner la parole» ou d'autoriser une participation qui serait «directe", mais a minima de questionner les cadres classiques de production et de diffusion de la recherche. Par ailleurs, les ateliers de cartographie, entre art et sciences humaines, ouvrent un axe critique sur les limites disciplinaires des pratiques de recherche et des savoirs produits, en souhaitant devenir un laboratoire d'expérimentation de pratiques de recherche indisciplinaires.

\section{BIBLIOGRAPHIE}

AGIER M. (2011), Le couloir des exilés : Être étranger dans un monde commun, Paris, Éditions du Croquant, $117 \mathrm{p}$.

AMILHAT-SZARY A.-L. (2012), « Border art and the politics of art display », Journal of Borderlands Studies, 2012/27 (2), pp. 213-228

ARAB C. (2007), « La « Hrague ", ou comment les Marocains brûlent les frontières ", Hommes et Migrations, 2007/1266, pp. 82-94

BENNAFLA K., PERALDI M. (2008), «Introduction. Frontières et logiques de passage : l'ordinaire des transgressions ", Cultures \& Conflits, 2008/72, pp. 7-12

BERNADRIE-TAHIR N., SCHMOLL C. (2012), « La voix des chercheur(-e)s et la parole des migrants. Ce que les coulisses du terrain maltais nous enseignent ", Carnets de Géographes, 2012/4, consulté le 1er septembre 2013, http://www.carnetsdegeographes.org/carnets_terrain/ terrain_04_01_Bernardie_Tahir_Schmoll.php

BESSE J.-M. 2010, « Cartographies », Les Carnets du paysage, 2010/20, pp. 5-9

CHOPLIN A., PLIEZ O. (2011), « De la difficulté de cartographier l'espace saharo-sahélien », Mappemonde, 2010/103, http://mappemonde.mgm.fr/num31/intro/intro2.html, consulté le 1er septembre 2013

CLOCHARD O., RESEAU MIGREUROP $(2009,2012)$, Atlas des migrants en Europe. Géographie critique des politiques migratoires, Paris, Armand Colin, 144p.

COUNTER CARTOGRAPHIES COLLECTIVE, DALTON C., MASON-DEESE L. (2012), « Counter (Mapping) Actions : Mapping as Militant Research », ACME : An International E-Journal for Critical Geographies, 2012/11 (3), pp. 439-466

COSGROVE D. (éd.) (1999), Mappings, Londres, Reaktion Books, 320p. 
COSGROVE D. (2001), Apollo's Eye : A Cartographic Genealogy of the Earth in the Western Imagination, Baltimore, The Johns Hopkins University Press, 331p.

CRAMPTON J. (2009), « Cartography : performative, participatory, political », Progress in Human Geography, 2009/33, pp. 840-848

DELIGNY F. (2007), Oeuvres, L’Arachnéen, Paris, 1848p.

FARET L. (2002), Les territoires de la mobilité. Espaces migratoires et Communautés transnationales entre le Mexique et les États-Unis, Paris, CNRS Éditions, 384p.

FARET L., CORTES G. (2009), Les circulations transnationales. Lire les turbulences migratoires contemporaines, Paris, Armand Colin, 208p.

FASSIN D., KOBELINKSY C. (2012), « Comment on juge l'asile. L'institution comme agent moral », Revue française de sociologie, 2012/4 (53), pp. 657-688

GRISON L. (2002), Figures fertiles : essai sur les figures géographiques dans l'art occidental, Nîmes, Éditions Jacqueline Chambon, 268p.

LAROUCHE J.-M., PIRON F. (éds.) (2010), « Responsabilité sociale et éthique de la recherche », Éthique Publique, 2010/12 (1), consulté le 1er septembre 2013, http:// ethiquepublique.revues.org/84

LOTY L. (2005), « Pour l'indisciplinarité », in Douthwaite J., Vidal M. (éds.), The Interdisciplinary Century ; Tensions and convergences in 18th-century Art, History and Literature, Oxford, Studies on Voltaire and the Eighteenth Century, Voltaire Foundation, pp. 245-259

LYNCH K. (1998), L'image de la cité, Paris, Dunod, 221p.

PIAN A. (2009), Aux nouvelles frontières de l'Europe : L'aventure incertaine des Sénégalais au Maroc, Paris, La Dispute, 237p.

PLIEZ O. (2011), Les cités du désert. Des villes sahariennes aux saharatowns, Marseille-Toulouse, Presses Universitaires du Mirail, 161p.

ROGOFF I. (2000), Terra Infirma : Geography's Visual Culture, London, Routledge, 216 p.

SPIVAK G. S. (2006), Les subalternes peuvent-elles parler ?, Paris, Éditions Amsterdam, 110p.

TARRIUS A., MISSAOUI L., QACHA F. (2013), Transmigrants et nouveaux étrangers, MarseilleToulouse, Presses Universitaires du Mirail, 200p.

TIBERGHIEN G. A. (2010), « Poétique et rhétorique de la carte dans l'art contemporain », L'Espace géographique, 2010/39, pp. 197-210

VOLVEY A. (2007), « Land arts. Les fabriques spatiales de l'art contemporain », Travaux de l'Institut de Géographie de Reims, 2007/129-130, pp. 3-25

WOOD D. (1992), The Power of Maps, New York, The Guilford Press, 248p.

WOOD D. (2010), Rethinking the Power of Maps, New York, The Guilford Press, 335p.

\section{NOTES}

1. Pendant les premières séances d'ateliers, les chercheures ont proposé d'évoquer les "voyages" plutôt que les "trajectoires migratoires" ou les "migrations" de chacun.e. Il s'agissait de trouver d'autres catégories que celles employées habituellement par les associations et les administrations pour commencer à élaborer des narrations alternatives. Alors que les 
administrations s'adressent à des "migrants" et plus particulièrement à des "demandeurs d'asile" qui doivent prouver que leur "migration" est bien "forcée" (Fassin, Kobelinsky, 2012), en accord avec les critères établis par la Convention de Genève, nous avons évoqué des "voyages”, vécus par des "voyageur.se.s".

2. "Demandeur d'asile" est un statut administratif octroyé en France aux personnes dont la demande d'asile a été enregistrée en préfecture et qui est examinée par l'office Français de Protection des Réfugiés et des Apatrides (OFPRA).

3. Suite à une demande d'asile, deux formes de protection sont possibles : le statut de réfugié et la protection subsidiaire qui n'ouvrent pas aux mêmes droits. Pour une explication des conditions d'obtention, se référer à http://www.france-terre-asile.org/demande-dasile/statutde-refugie.

4. Le texte non-encadré est écrit par Sarah Mekdjian et Anne-Laure Amilhat-Szary. Les textes encadrés et la figure 3 sont écrits par Marie Moreau, Mabeye Deme, Gladeema Nasruddiun et Lauriane Houbey respoectivement. Les textes encadrés et la figure 3 sont signés dans le texte par leurs auteurs.

5. Au total, six personnes ont co-encadré les ateliers: Sarah Mekdjian et Anne-Laure AmilhatSzary, enseignantes-chercheuses en géographie à l'Université de Grenoble et au laboratoire PACTE, membres du projet de recherche européen EUBorderscapes (FP7), Marie Moreau, Lauriane Houbey et Fabien Fischer, artistes, membres de l'association ex.C.es, Mabeye Deme, photographe indépendant, et Coralie Guillemin, bénévole à l'ADA, engagée comme ingénieure de recherche le temps du projet. Pour mener à bien cette expérience, nous avons bénéficié des fonds $\mathrm{du}$ projet européen de recherche EUBorderscapes, dont l'axe 10 porte sur les liens entre franchissements frontaliers et productions culturelles (http://www.euborderscapes.eu), coordonné pour l'université de Grenoble par Anne-Laure Amilhat-Szary.

6. Cette association, financée par des subventions de l'État et des collectivités territoriales principalement, est l'une des deux associations grenobloises qui accompagnent les personnes qui demandent l'asile dans leur procédure administrative (http://ada-grenoble.org/).

2. Exposition Géographies Intérieures de Marie Moreau (exposition d'Atlas Local \#1) et Lauriane Houbey. Laboratoire d'Art Aujourd'hui. Grenoble France. 2012

7. Le collectif Art-Sciences-Technologie «Anti-Atlas des frontières » (http://www.antiatlas.net/) diffuse de nombreuses œuvres et productions de contre-cartographie sur les franchissements frontaliers. Les travaux réalisés pendant nos ateliers ont d'ailleurs été diffusés dans le cadre d'une exposition collective au Musée des Tapisseries à Aix-en-Provence du 1er Octobre au 3 Novembre 2013 (http://www.antiatlas.net/blog/2013/09/20/crossing-maps-cartographiestraverses/in English: http://www.antiatlas.net/en/2013/09/21/crossing-maps-fabien-fischerlauriane-houbey-sarah-mekdjian-et-anne-laure-amilhat-szary-marie-moreau/). Au sujet de la contre-cartographie, voir Counter Cartographies Collective (http://countercartographies.org), Dalton, Craig, Mason-Deese, Liz, 2012.

8. L'art ne constituant pas une «discipline», au sens d'une spécialisation et d'une institutionnalisation des savoirs scientifiques, la mise en relation des sciences et de l'art ne peut être qualifiée de pluri- ou trans-disciplinaire. Laurent Loty, à partir d'une histoire de la notion de discipline, explique l'intérêt de ce qu'il appelle «l'indisciplinarité »: "à l'intérêt d'une indisciplinarité des objets d'étude s'ajoute celui d'une indisciplinarité des démarches employées. L'inscription des recherches dans des disciplines spécialisées est susceptible d'invalider ou même de rendre impensables des questionnements qui surgiraient précisément du refus des objets et méthodes reconnus par les disciplines » (Loty, 2005, p. 252).

9. Le 20 juin 2013, les cartes réalisées ont été exposées dans un centre social de la municipalité de Grenoble où, à l'intitiative de plusieurs demandeurs d'asile et associations grenobloises, dont l'ADA, avait été organisée la Journée Mondiale des Réfugiés. A l'occasion de cette exposition, nous 
sommes revenus collectivement sur l'organisation des ateliers et notamment leur financement par un fonds de recherche européen.

10. Cf. notamment http://www.terraeco.net/A-Grenoble-les-cartes-des,50258.html

11. Ce projet a été financé dans le cadre du programme européen EUBorderscapes (http:// www.euborderscapes.eu/)

\section{RÉSUMÉS}

Entre mai et juin 2013 s'est tenu à Grenoble un atelier de cartographie participatif et expérimental, à la croisée des sciences humaines et de l'art. Douze voyageurs, alors demandeurs d'asile ou réfugiés, trois artistes et deux géographes se sont réunis pour aborder la cartographie comme technique créative de relevé d'expériences. Les cartes produites avec et par les participant.e.s évoquent des souvenirs d'entre-deux migratoires et de franchissements frontaliers. Cette expérience participative cherche à se démarquer d'un positivisme narratif des itinéraires migratoires -caractéristique notamment des administrations en charge du droit d'asile-, en mobilisant des outils de médiation visuels et artistiques. Les acteurs de ce projet reviennent ici sur les intentions de construire un terrain de recherche indisciplinaire, fondé sur une pratique cartographique critique et créative. Les cartes ont constitué des entre-deux méthodologiques au service des relations entre les participants, tandis que s'élaboraient des récits référentiels et non-référentiels des entre-deux migratoires.

Over a two-month period, twice a week, in May and June 2013, two researchers, three contemporary artists, twelve refugees and a photographer met together in Grenoble, France. The workshops were intended to create encounters around mapping performances, to produce alternative narratives on migrations, experiences of socio-spatial exclusion and inbetweenness.From a methodological point of view, we tried not to reproduce some ethical biases of the ethnographic life-story method by using mapping. The maps were a framework within which collective and individual interactions evolved, so that they were a third party in the relationships build up during the workshops. This critical mapping experience raises methodological and ethical questions as to the conditions on which the migrants, researchers and artists took part, on the alternative narrative methods used, and on the scientific, aesthetic and political uses of the maps produced.

\section{INDEX}

Thèmes : Carnets de terrain

\section{AUTEURS}

\section{SARAH MEKDJIAN}

Université Pierre-Mendès-France, Grenoble II

Laboratoire PACTE 
Programme EUBorderscapes (FP7)

Géographie

sarah.mekdjian@upmf-grenoble.fr

ANNE-LAURE AMILHAT-SZARY

Université Joseph Fourier, Grenoble I

Laboratoire PACTE

Programme EUBorderscapes (FP7)

Géographie

anne-laure.amilhat@ujf-grenoble.fr

\section{MARIE MOREAU}

Association L'ex.C.es

Artiste plasticienne

\section{GLADEEMA NASRUDDIN}

Participant des ateliers de cartographie

Demandeur d'asile

\section{MABEYE DEME}

Photographe indépendant

mabeyedeme@gmail.com

\section{LAURIANE HOUBEY}

Association L'ex.C.es

Artiste chorégraphe, plasticienne

\section{CORALIE GUILLEMIN}

Ingénieure de recherche contractuelle

Laboratoire PACTE, Programme EUBorderscapes (FP 7) 